\title{
Inhibitory effects of paeoniflorin on lysophosphatidylcholine- induced inflammatory factor production in human umbilical vein endothelial cells
}

\author{
JIAN-ZHE LI ${ }^{1 *}$, JIAN-HUA WU ${ }^{2 *}$, SHU-YI YU ${ }^{3}$, QING-RUI SHAO ${ }^{1}$ and XIAO-MIN DONG ${ }^{2}$ \\ ${ }^{1}$ Department of Pharmacy, Ruikang Hospital, Guangxi University of Traditional Chinese Medicine, Nanning, Guangxi 530011; \\ ${ }^{2}$ Department of Pharmacy, Zhongnan Hospital of Wuhan University, Wuhan, Hubei 430071; \\ ${ }^{3}$ Modern Analysis and Testing Center, Central South University, Changsha, Hunan 410078, P.R. China
}

Received October 15, 2012; Accepted November 29, 2012

DOI: $10.3892 / \mathrm{ijmm} .2012 .1211$

\begin{abstract}
Lysophosphatidylcholine (LPC) plays an important role in atherosclerosis through initiation of endothelial inflammation response. Paeoniflorin (PEF), isolated from the dry root of Paeonia, has been reported to exert an anti-inflammatory effect, but the exact mechanism is not fully understood. The aim of this study was to investigate the inhibitory effects of PEF on LPC-induced inflammatory factor production and the underlying mechanisms. In human umbilical vein endothelial cells (HUVECs), different concentrations $(1,10$ or $100 \mu \mathrm{mol} / \mathrm{l})$ of PEF were added $2 \mathrm{~h}$ prior to exposure to LPC $(10 \mathrm{mg} / \mathrm{l})$ for $24 \mathrm{~h}$. The results showed that PEF significantly inhibited LPC-induced inflammatory factor production. In addition, PEF was also able to suppress the enhanced high mobility group box-1 (HMGB1) expression and release, upregulated expression of receptor for advanced glycation end product (RAGE), Toll-like receptor (TLR)-2 and TLR-4, and increased nuclear factor $-\kappa \mathrm{B}(\mathrm{NF}-\kappa \mathrm{B})$ activity induced by LPC. Our results suggest that PEF suppresses LPC-induced inflammatory factor production through inhibition of the HMGB1-RAGE/ TLR-2/TLR-4-NF- $\kappa$ B pathway.
\end{abstract}

\section{Introduction}

It is well recognized that atherosclerosis is a chronic inflammatory disease (1). Vascular inflammatory response plays an important role in the onset, development and evolution of atherosclerosis (2). Many reports have demonstrated that vascular

Correspondence to: Dr Jian-Zhe Li, Department of Pharmacy, Ruikang Hospital, Guangxi University of Traditional Chinese Medicine, Hua-Dong Road 10, Nanning 530011, P.R. China

E-mail: jianzhe_li@yahoo.com

*Contributed equally

Key words: paeoniflorin, lysophosphatidylcholine, inflammatory factors, high mobility group box-1, human umbilical vein endothelial cells inflammatory response is closely associated with endothelial dysfunction $(3,4)$. Endothelial cells can release several inflammatory factors in response to a variety of harmful stimulations including increased lysophosphatidylcholine (LPC) (5). LPC is the major phospholipid component of oxidized low-density lipoprotein (ox-LDL) (6), and it is well known for its ability to mimic the effects of ox-LDL (7). Increased plasma ox-LDL is an independent risk factor in atherosclerosis and plays a key role in initiation of the inflammatory response $(8,9)$. Recently, it has been reported that ox-LDL-induced inflammation in endothelial cells is related to stimulation of high mobility group box-1 (HMGB1) release (10).

HMGB1 is a non-histone DNA-binding nuclear protein that can be positively released from immune-activated cells or passively released from necrotic cells (11). There is growing evidence that extracellular HMGB1 is a very potent proinflammatory mediator $(12,13)$. HMGB1 can stimulate the expression and release of numerous inflammatory factors, such as tumor necrosis factor- $\alpha(\mathrm{TNF}-\alpha)$, interleukins, intercellular adhesion molecule-1 (ICAM-1) and selectins (14,15). It has been reported that the pro-inflammatory effect of HMGB1 is mediated mainly through binding to its specific receptors, including receptor for advanced glycation endproduct (RAGE), Toll-like receptor (TLR)-2 and TLR-4 (16). The binding of HMGB1 to its specific receptors ultimately results in the activation of nuclear factor- $\kappa \mathrm{B}(\mathrm{NF}-\kappa \mathrm{B})$, and then upregulation of inflammatory factor expression and release $(17,18)$. Therefore, the HMGB1-RAGE/TLR-2/TLR-4-NF- $\kappa$ B pathway may be an important inflammatory signaling pathway.

Paeoniflorin (PEF), a monoterpene glucoside, is the primary active ingredient extracted from the dry root of Paeonia, which is a traditional Chinese herbal medicine extensively used in China for more than 1000 years to treat numerous diseases, such as virus hepatitis, anemia, systemic lupus erythematosus and gynecological diseases (19). In recent years, it has been reported that PEF exhibits anti-inflammatory properties. In Sprague-Dawley rats, both pretreatment and post-treatment with PEF alleviated ischemia/reperfusion-induced cerebral injury, at least in part, through inhibition of inflammatory factor production (20). In addition, PEF was able to protect against lipopolysaccharide-induced acute lung injury in mice 
by alleviating inflammatory cell infiltration and microvascular permeability (21). However, the exact anti-inflammatory mechanisms of PEF remain unclear.

In the present study, we investigated whether PEF has an inhibitory effect on LPC-induced inflammatory factor production using cultured human umbilical vein endothelial cells (HUVECs), and whether the anti-inflammatory effect is related to the inhibition of the HMGB1-RAGE/TLR-2/TLR-4$\mathrm{NF}-\kappa \mathrm{B}$ pathway.

\section{Materials and methods}

Materials. HUVECs were obtained from the Tumor Research Institute of Peking University (Peking, China). PEF (purity $\geq 98 \%$ ) was purchased from the Yangling Dongke Pharmaceutical Division (Shanxi, China). LPC was purchased from Sigma-Aldrich (St. Louis, MO, USA). Dulbecco's modified Eagle's medium (DMEM) and fetal bovine serum (FBS) were provided by Gibco-BRL (Grand Island, NY, USA). TRIzol reagent was a product of Invitrogen Corp. (Carlsbad, CA, USA). The First Strand cDNA Synthesis kit was purchased from MBI Fermentas Inc. (Vilnius, Lithuania). RAGE, TLR-2, TLR-4 and $\beta$-actin antibodies were from Abcam (Cambridge, UK). 3-(4,5-Dimethylthiazol-2-yl)-2,5-diphenyltetrazolium bromide (MTT) was from Beyotime Biotechnology (Jiangsu, China). ICAM-1, MCP-1, IL-6, TNF- $\alpha$, HMGB1 and NF- $\kappa$ Bp65 ELISA kits were obtained from the Nanjing Jiancheng Bioengineering Institute (Nanjing, China).

Cell culture and treatment. HUVECs were cultured in DMEM containing $10 \%(\mathrm{v} / \mathrm{v}) \mathrm{FBS}, 100 \mathrm{U} / \mathrm{ml}$ penicillin and $100 \mu \mathrm{g} /$ $\mathrm{ml}$ streptomycin in a humidified atmosphere of $5 \% \mathrm{CO}_{2}$ at $37^{\circ} \mathrm{C}$. Cells were passaged by trypsinization and seeded at $\sim 10^{5}$ cells $/ \mathrm{ml}$. When cells reached $80 \%$ confluence, the culture medium was replaced with serum-free medium for $24 \mathrm{~h}$, and then cells were pretreated with various concentrations $(1,10$ or $100 \mu \mathrm{mol} / \mathrm{l})$ of PEF $2 \mathrm{~h}$ prior to exposure to LPC (10 mg/l) for $24 \mathrm{~h}$. LPC was dissolved in ethanol. The final concentration of ethanol was less than $0.1 \%$ (v/v). Cells used in the experiments were from 5 to 8 passages.

Cell viability assay. MTT was used to determine cell viability. Briefly, HUVECs were seeded at a density of $1 \times 10^{4}$ cells/well in a 96-well culture plate. After drug treatment, the cells were washed twice with PBS to remove the medium, and $10 \mu \mathrm{l}$ of MTT $(0.5 \mathrm{mg} / \mathrm{ml})$ was added to each well and incubated for an additional $4 \mathrm{~h}$ at $37^{\circ} \mathrm{C}$. Subsequently, $100 \mu \mathrm{l}$ of dimethy sulfoxide (DMSO) was added to dissolve the MTT, and the absorbance at $490 \mathrm{~nm}$ was read on a microplate reader. Data are expressed as a percentage of the control, which was considered to be $100 \%$ viable.

ELISA. After drug treatment, cell culture supernatants were collected and centrifuged at 3,000 x g for $10 \mathrm{~min}$ to remove debris. The nuclear lysates were prepared as described by Wu et al (22). Briefly, cells were washed with cold PBS and then scraped from the well. Cells were treated with hypotonic buffer and centrifuged. The pellet was collected and treated with cell extraction buffer, vortexed, centrifuged and the supernatants (nuclear lysates) were stored at $-70^{\circ} \mathrm{C}$. The concentrations of ICAM-1, MCP-1, IL-6, TNF- $\alpha$ and HMGB1 in cell culture supernatants and NF- $\kappa$ Bp65 in nuclear lysates were determined by commercially available ELISA kits according to the manufacturer's instructions.

Quantitative real-time PCR. Total RNA was isolated from HUVECs using TRIzol reagent and quantified by measuring the optical density at $260 \mathrm{~nm}$. cDNA was synthesized from $1 \mu \mathrm{g}$ of total RNA, which was the used for quantitative real-time PCR. Quantitative analysis of mRNA expression was performed using the ABI 7300 real-time PCR system with the Power SYBR-Green PCR Master Mix kit. PCR primers were as follows: HMGB1 (forward, 5'-ATGTTGCGAAGAAACTGG-3' and reverse, 5'-TTCAGCCTTGACAACTCC-3'); RAGE (forward, 5'-AAG CCCCTGGTGCCTAATGAG-3' and reverse, 5'-CACCAATT GGACCTCCTCCA-3'); TLR-2 (forward, 5'-ATCCTCCAATC AGGCTTCTCT-3' and reverse, 5'-ACACCTCTGTAGGTCAC TGTTG-3'); TLR-4 (forward, 5'-ATATTGACAGGAAACCCC ATCCA-3' and reverse, 5'-AGAGAGATTGAGTAGGGGCAT TT-3'); GAPDH (forward, 5'-CAATGACCCCTTCATTGA-3' and reverse, 5'-GACAAGCTTCCCGTTCTCAG-3'). The PCR amplification profiles consisted of denaturation at $95^{\circ} \mathrm{C}$ for $10 \mathrm{~min}$, followed by 40 cycles of denaturation at $95^{\circ} \mathrm{C}$ for $15 \mathrm{sec}$ and annealing at $60^{\circ} \mathrm{C}$ for $60 \mathrm{sec}$. All amplification reactions for each sample were carried out in triplicates, and the relative expression values were normalized to the expression value of GAPDH.

Western blot analysis. The protein expression of RAGE, TLR-2 and TLR-4 was determined by western blotting. Briefly, after drug treatment, cells were lysed with ice-cold lysis buffer [0.33 mol/1 Tris/HCl, $10 \%$ SDS (wt/vol), $40 \%$ glycerol (vol/vol) and $50 \mathrm{mmol} / \mathrm{l}$ DTT containing bromophenol blue], and the protein content of the lysates was measured using the bicinchoninic acid (BCA) method. Equal amounts of protein were separated by $10 \%$ SDS-PAGE and transferred to a nitrocellulose membrane. After being blocked with TBST containing $5 \%$ bovine serum albumin, the membranes were incubated with the primary antibody for RAGE, TLR-2, TLR- 4 and $\beta$-actin overnight at $4^{\circ} \mathrm{C}$, and then incubated with the corresponding horseradish peroxidase-conjugated secondary antibody at room temperature for $1 \mathrm{~h}$. The protein bands were quantitated by video densitometry, and the results were normalized to $\beta$-actin expression.

Statistical analysis. Data are expressed as means \pm SEM. All values were analyzed by analysis of variance followed by the Student-Newman-Keuls test. $\mathrm{P}<0.05$ was regarded as indicative of statistical significance.

\section{Results}

Effect of PEF on cell viability induced by LPC. MTT assay showed that treatment of HUVECs with LPC $(10 \mathrm{mg} / \mathrm{l})$ for $24 \mathrm{~h}$ significantly decreased the cell viability. Pretreatment with PEF $2 \mathrm{~h}$ prior to exposure to LPC concentration dependently inhibited the decrease in cell viability induced by LPC. However, PEF alone had no effect on cell viability (Fig. 1).

Effect of PEF on inflammatory factor production induced by $L P C$. ELISA showed that treatment with LPC significantly 


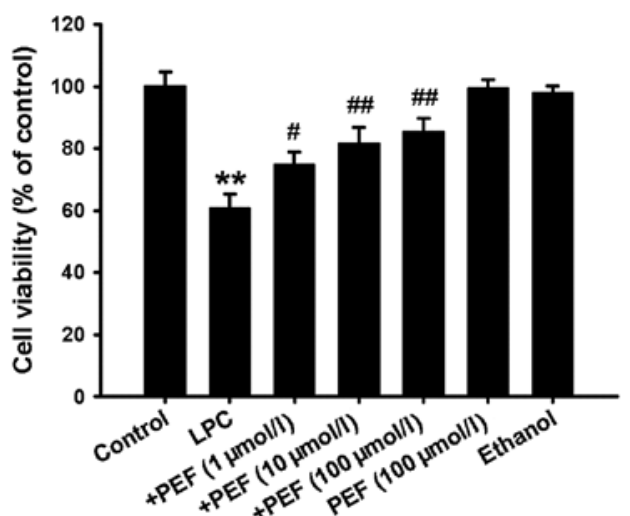

Figure 1. Effect of PEF on LPC-induced cell viability in HUVECs. Data are expressed as means \pm SEM and represent three independent experiments. ${ }^{* *} \mathrm{P}<0.01$ vs. control; ${ }^{\#} \mathrm{P}<0.05,{ }^{\# \#} \mathrm{P}<0.01$ vs. $\mathrm{LPC}$.
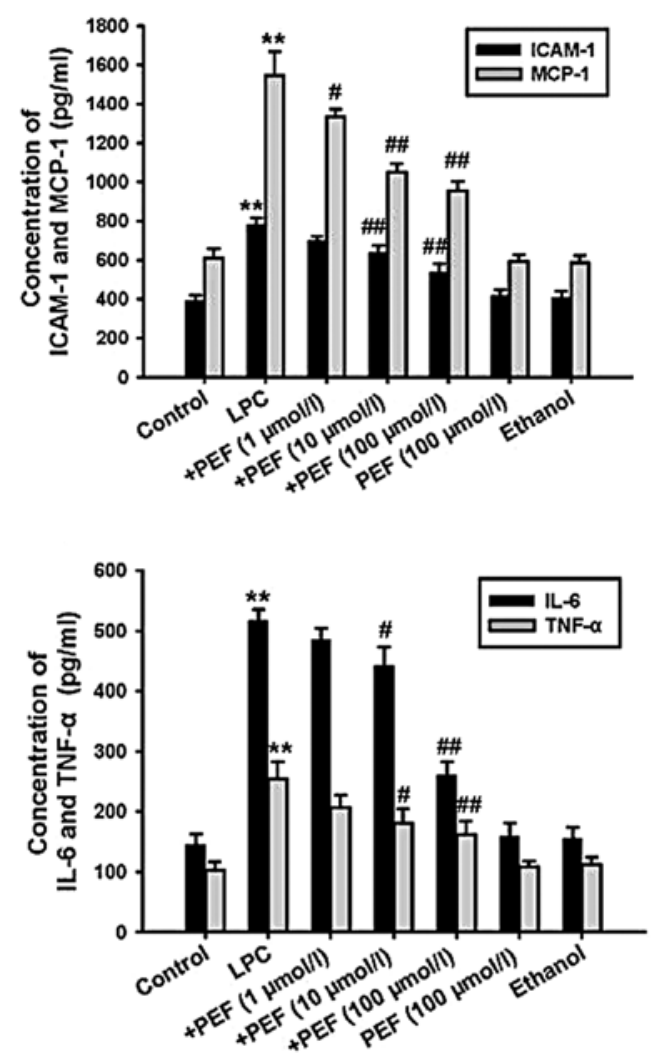

Figure 2. Effect of PEF on LPC-induced inflammatory factor production in HUVECs. Data are expressed as means \pm SEM and represent three independent experiments. ${ }^{* *} \mathrm{P}<0.01$ vs. control; ${ }^{*} \mathrm{P}<0.05,{ }^{\# \#} \mathrm{P}<0.01$ vs. LPC.

increased the concentration of ICAM-1, MCP-1, IL- 6 and TNF- $\alpha$ in cell culture supernatants. This effect of LPC was markedly attenuated by pretreatment with PEF in a concentration-dependent manner. However, PEF alone had no effect on inflammatory factor production (Fig. 2).

Effect of PEF on HMGB1 expression and release induced by $L P C$. Real-time PCR analysis showed that HMGB1 mRNA expression was significantly upregulated in LPC-treated HUVECs. Consistent with this result, treatment with LPC

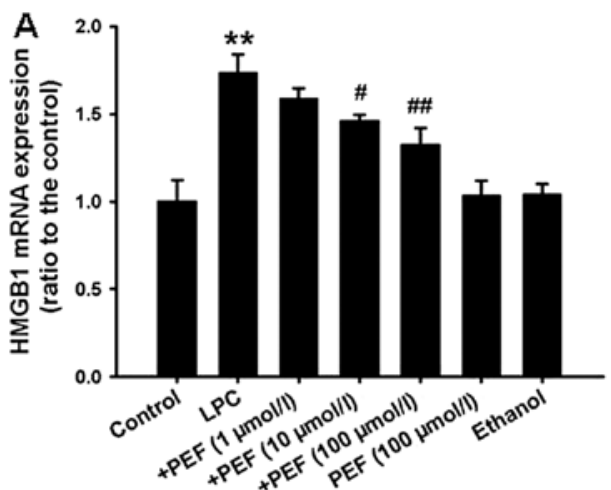

B

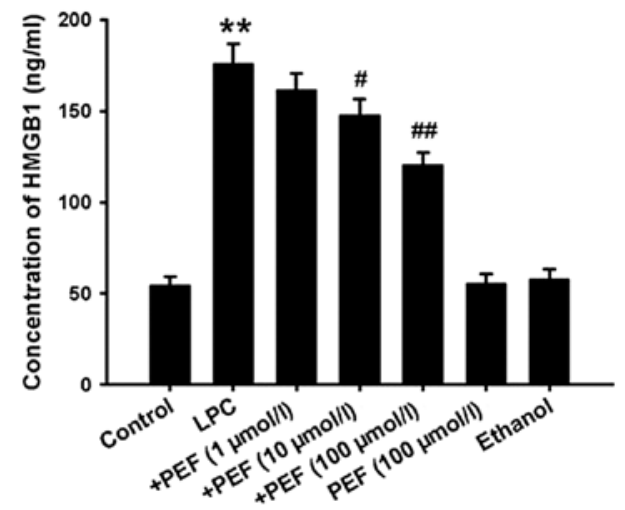

Figure 3. Effect of PEF on LPC-induced HMGB1 mRNA (A) expression and (B) release in HUVECs. Data are expressed as means \pm SEM and represent three independent experiments. ${ }^{* *} \mathrm{P}<0.01$ vs. control; ${ }^{\#} \mathrm{P}<0.05,{ }^{\# \#} \mathrm{P}<0.01$ vs. LPC.

markedly increased the concentration of HMGB1 in cell culture supernatants. However, these effects of LPC were reversed by pretreatment with $\mathrm{PEF}$ in a concentration-dependent manner. PEF alone had no effect on HMGB1 mRNA expression and release (Fig. 3).

Effect of PEF on the expression of HMGB1 receptors induced by $L P C$. The mRNA expression of RAGE, TLR-2 and TLR-4 was significantly upregulated in HUVECs treated with LPC compared with the controls (Fig. 4A). Consistent with the mRNA expression, treatment with LPC significantly increased the protein expression of RAGE, TLR-2 and TLR-4 (Fig. 4B and C). These effects were attenuated by pretreatment with different concentrations of PEF.

Effect of PEF on NF- $\kappa$ B activation induced by LPC. Since activation of NF- $\kappa B$ is essential to inflammatory factor production, we therefore detected NF- $\mathrm{kB}$ activity. Treatment of HUVECs with LPC significantly increased NF- $\kappa B$ activity, which was indicated by the increased optical density of NF- $\mathrm{KBp} 65$ in the nuclear lysates (Fig. 5). This effect was markedly inhibited by pretreatment with different concentrations of PEF.

\section{Discussion}

In the present study, we investigated the inhibitory effect of PEF on LPC-induced inflammatory factor production and 

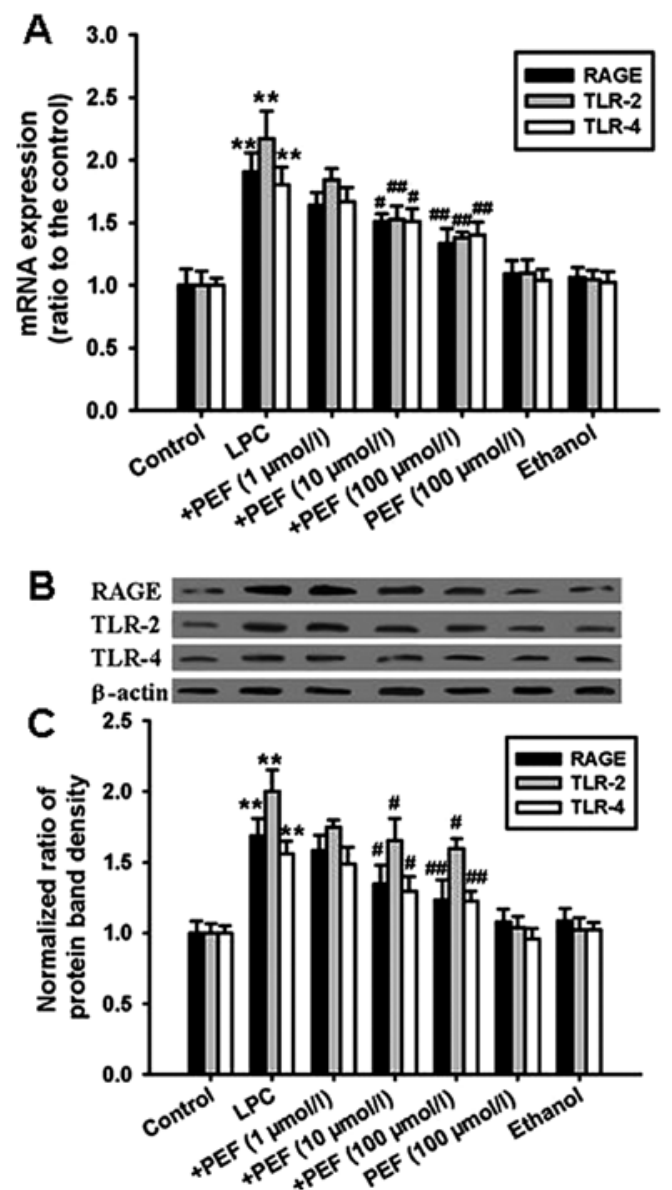

Figure 4. Effect of PEF on LPC-induced RAGE, TLR-2 and TLR-4 expression in HUVECs. (A) mRNA expression of RAGE, TLR-2 and TLR-4 by real-time PCR. (B) Representative image of RAGE, TLR-2 and TLR-4 protein expression by western blotting. (C) Optical density of protein bands. Data are expressed as means \pm SEM and represent three independent experiments. ${ }^{* *} \mathrm{P}<0.01$ vs. control; ${ }^{\#} \mathrm{P}<0.05,{ }^{\# \#} \mathrm{P}<0.01$ vs. $\mathrm{LPC}$.

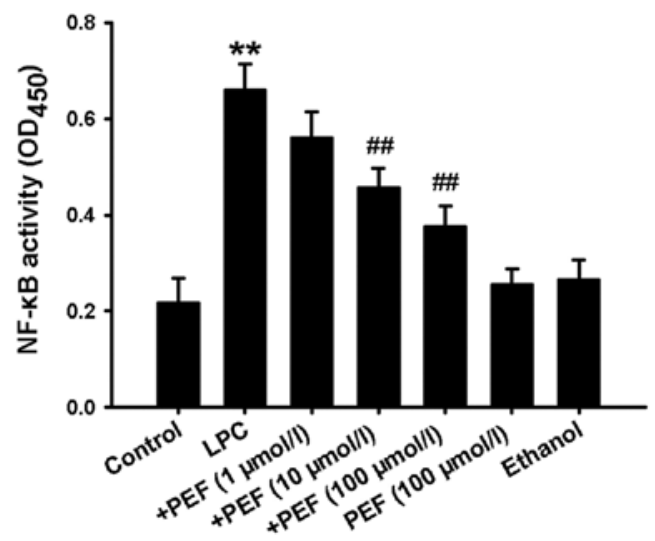

Figure 5. Effect of PEF on LPC-induced NF- $\kappa B$ activation in HUVECs. Data are expressed as the mean optical density (OD) at $450 \mathrm{~nm} \pm$ SEM and represents three independent experiments. ${ }^{* *} \mathrm{P}<0.01$ vs. control; ${ }^{\# \#} \mathrm{P}<0.01$ vs. LPC.

the underlying mechanisms in HUVECs. The results showed that pretreatment with PEF was able to inhibit LPC-induced inflammatory factor production in a concentration-dependent manner, which was related to inhibition of HMGB1 expres- sion and release, downregulation of RAGE, TLR-2 and TLR-4 expression, and decrease in NF- $\mathrm{kB}$ activity.

Vascular inflammatory response is well known to play a crucial role in all stages of atherosclerosis from the nascent lesion to acute coronary syndromes (23). Inhibition of the inflammatory response is a potential strategy to prevent the development of atherosclerosis $(8,9,24)$. LPC is the major bioactive lipid component of ox-LDL and is implicated as an important factor in the atherogenic activity of ox-LDL (25). Many studies have indicated that the atherogenic effect of LPC is closely related to the inflammatory response. Gonçalves et al (26) reported that an increased level of LPC plays a key role in human atherosclerotic plaque inflammation. In addition, LPC was found to significantly increase the adherence of monocytes to endothelial cells and upregulate the expression of ICAM-1 and vascular cell adhesion molecule-1 (VCAM-1), which are both biomarkers in inflammatory progression (5). Therefore, inhibition of LPC-induced inflammation may be a promising approach by which to prevent the development of atherosclerosis.

HMGB1, also named amphoterin, is a highly conserved chromatin binding protein that is expressed in multiple cell types including endothelial cells (27). HMGB1 overexpression and release can initiate and amplify the inflammatory response (28). Recently, extracellular HMGB1 was identified as a potent pro-inflammatory cytokine (16) and plays a decisive role in the mediation of inflammatory responses (29). Therefore, HMGB1 is recognized as a potential therapeutic target in inflammatory diseases (28). As previously mentioned, HMGB1 interacts with RAGE, TLR-2 and TLR-4, the cell surface receptors of HMGB1, to amplify inflammatory responses by activating NF- $\mathrm{kB}$ and then inducing inflammatory factor expression and release (30). Therefore, the HMGB1-RAGE/TLR-2/TLR-4-NF-кB pathway may be an important inflammatory signaling pathway.

PEF, a monoterpene glucoside isolated from the dry root of Paeonia, has been reported to have multiple beneficial effects, such as the lowering of cholesterol levels, anti-platelet agglutination and neuroprotective effects (31-33). Recently, there is growing evidence that PEF exerts an anti-inflammatory effect in animal models of collagen-induced arthritis (34), ischemia/ reperfusion-induced cerebral injury (20), lipopolysaccharideinduced acute lung injury (21) and liver inflammatory reactions (35). However, the exact anti-inflammatory mechanisms of PEF are still unclear. Since PEF has anti-inflammatory properties and the HMGB1-RAGE/TLR-2/TLR-4-NF- $\mathrm{BB}$ pathway is an important inflammatory signaling pathway, we therefore hypothesized that PEF may be able to inhibit LPC-induced inflammatory factor production in HUVECs, and the mechanism may be associated with inhibition of the HMGB1-RAGE/ TLR-2/TLR-4-NF- $\mathrm{kB}$ signaling pathway. In the present study, we found that pretreatment of PEF significantly inhibited LPC-induced inflammatory factor production concomitantly with decreased expression and release of HMGB1, downregulated mRNA and protein expression of RAGE, TLR-2 and TLR-4, and decreased NF- $\mathrm{kB}$ activity. These results confirmed our hypothesis.

In summary, the present study demonstrated that PEF was able to inhibit LPC-induced inflammatory factor production, which was related to inhibition of the HMGB1-RAGE/TLR-2/ TLR-4-NF- $\mathrm{BB}$ signaling pathway. 


\section{Acknowledgements}

This study was supported by the National Nature Science Foundation of China (81101476 to S.Y.Y.).

\section{References}

1. Ross R: Atherosclerosis is an inflammatory disease. Am Heart J 138: S419-S420, 1999.

2. Lin R, Liu J, Gan W and Yang G: C-reactive protein-induced expression of CD40-CD40L and the effect of lovastatin and fenofibrate on it in human vascular endothelial cells. Biol Pharm Bull 27: 1537-1543, 2004

3. Loppnow H, Buerke M, Werdan K and Rose-John S: Contribution of vascular cell-derived cytokines to innate and inflammatory pathways in atherogenesis. J Cell Mol Med 15: 484-500, 2011.

4. Sprague AH and Khalil RA: Inflammatory cytokines in vascular dysfunction and vascular disease. Biochem Pharmacol 78: 539-552, 2009.

5. Kang H, Yang PY and Rui YC: Adenoviral gene transfer of viral interleukin-10 protects cerebrovascular impairment induced by lysophosphatidylcholine. Eur J Pharmacol 580: 175-181, 2008.

6. Matsumoto T, Kobayashi T and Kamata K: Role of lysophosphatidylcholine (LPC) in atherosclerosis. Curr Med Chem 14 3209-3220, 2007.

7. Schaefer CA, Kuhlmann CR, Gast C, et al: Statins prevent oxidized low-density lipoprotein- and lysophosphatidylcholineinduced proliferation of human endothelial cells. Vascul Pharmacol 41: 67-73, 2004.

8. Toshima S, Hasegawa A, Kurabayashi M, et al: Circulating oxidized low density lipoprotein levels. A biochemical risk marker for coronary heart disease. Arterioscler Thromb Vasc Biol 20: 2243-2247, 2000

9. Mazière $\mathrm{C}$ and Mazière $\mathrm{JC}$ : Activation of transcription factors and gene expression by oxidized low-density lipoprotein. Free Radic Biol Med 46: 127-137, 2009.

10. Yu X, Xing C, Pan Y, Ma H, Zhang J and Li W: IGF-1 alleviates ox-LDL-induced inflammation via reducing HMGB1 release in HAECs. Acta Biochim Biophys Sin 44: 746-751, 2012.

11. Zhang J, Takahashi HK, Liu K, et al: Anti-high mobility group box-1 monoclonal antibody protects the blood-brain barrier from ischemia-induced disruption in rats. Stroke 42: 1420-1428, 2011.

12. Naglova $\mathrm{H}$ and Bucova M: HMGB1 and its physiological and pathological roles. Bratisl Lek Listy 113: 163-171, 2012.

13. Chen G, Ward MF, Sama AE and Wang H: Extracellular HMGB1 as a proinflammatory cytokine. $\mathbf{J}$ Interferon Cytokine Res 24 329-333, 2004.

14. Nadatani Y, Watanabe T, Tanigawa T, et al: High mobility group box 1 promotes small intestinal damage induced by nonsteroida anti-inflammatory drugs through Toll-like receptor 4 . Am J Pathol 181: 98-110, 2012.

15. Lee W, Kim TH, Ku SK, Min KJ, Lee HS, Kwon TK and Bae JS: Barrier protective effects of withaferin A in HMGB1-induced inflammatory responses in both cellular and animal models. Toxicol Appl Pharmacol 262: 91-98, 2012.

16. Lee W, Ku SK, Bae JW and Bae JS: Inhibitory effects of lycopene on HMGB1-mediated pro-inflammatory responses in both cellular and animal models. Food Chem Toxicol 50: 1826-1833, 2012.

17. Park JS, Svetkauskaite D, He Q, Kim JY, Strassheim D, Ishizaka A and Abraham E: Involvement of toll-like receptors 2 and 4 in cellular activation by high mobility group box 1 protein J Biol Chem 279: 7370-7377, 2004.

18. Luan ZG, Zhang H, Yang PT, Ma XC, Zhang C and Guo RX: HMGB1 activates nuclear factor- $\kappa \mathrm{B}$ signaling by RAGE and increases the production of TNF- $\alpha$ in human umbilical vein endothelial cells. Immunobiology 215: 956-962, 2010.
19. He DY and Dai SM: Anti-inflammatory and immunomodulatory effects of Paeonia lactiflora pall., a traditional Chinese herbal medicine. Front Pharmacol 2: 10, 2011.

20. Tang NY, Liu CH, Hsieh CT and Hsieh CL: The anti-inflammatory effect of paeoniflorin on cerebral infarction induced by ischemia-reperfusion injury in Sprague-Dawley rats. Am J Chin Med 38: 51-64, 2010

21. Zhou H, Bian D, Jiao X, Wei Z, Zhang H, Xia Y, He Y and Dai Y: Paeoniflorin protects against lipopolysaccharide-induced acute lung injury in mice by alleviating inflammatory cell infiltration and microvascular permeability. Inflamm Res 60: 981-990, 2011.

22. Wu WC, Hu DN, Gao HX, Chen M, Wang D, Rosen R and McCormick SA: Subtoxic levels hydrogen peroxide-induced production of interleukin- 6 by retinal pigment epithelial cells. Mol Vis 16: 1864-1873, 2010.

23. Wang HR, Li JJ, Huang CX and Jiang H: Fluvastatin inhibits the expression of tumor necrosis factor-a and activation of nuclear factor-kappaB in human endothelial cells stimulated by C-reactive protein. Clin Chim Acta 353: 53-60, 2005.

24. Barish GD, Yu RT, Karunasiri MS, et al: The Bcl6-SMRT/NCoR cistrome represses inflammation to attenuate atherosclerosis. Cell Metab 15: 554-562, 2012.

25. Schmitz G and Ruebsaamen K: Metabolism and atherogenic disease association of lysophosphatidylcholine. Atherosclerosis 208: 10-18, 2010.

26. Gonçalves I, Edsfeldt A, Ko NY, et al: Evidence supporting a key role of Lp-PLA2-generated lysophosphatidylcholine in human atherosclerotic plaque inflammation. Arterioscler Thromb Vasc Biol 32: 1505-1512, 2012.

27. Orlova VV, Choi EY, Xie C, et al: A novel pathway of HMGB1-mediated inflammatory cell recruitment that requires Mac-1-integrin. EMBO J 26: 1129-1139, 2007.

28. Gao M, Hu Z, Zheng Y, Zeng Y, Shen X, Zhong D and He F: Peroxisome proliferator-activated receptor $\gamma$ agonist troglitazone inhibits high mobility group box 1 expression in endothelial cells via suppressing transcriptional activity of nuclear factor $\kappa \mathrm{B}$ and activator protein 1. Shock 36: 228-234, 2011.

29. Kim TH, Ku SK, Lee T and Bae JS: Vascular barrier protective effects of phlorotannins on HMGB1-mediated proinflammatory responses in vitro and in vivo. Food Chem Toxicol 50: 2188-2195, 2012.

30. Yang EJ, Lee W, Ku SK, Song KS and Bae JS: Anti-inflammatory activities of oleanolic acid on HMGB1 activated HUVECs. Food Chem Toxicol 50: 1288-1294, 2012.

31. Yang HO, Ko WK, Kim JY and Ro HS: Paeoniflorin: an antihyperlipidemic agent from Paeonia lactiflora. Fitoterapia 75: 45-49, 2004.

32. Koo YK, Kim JM, Koo JY, Kang SS, Bae K, Kim YS, Chung JH and Yun-Choi HS: Platelet anti-aggregatory and blood anticoagulant effects of compounds isolated from Paeonia lactiflora and Paeonia suffruticosa. Pharmazie 65: 624-628, 2010.

33. Mao QQ, Zhong XM, Qiu FM, Li ZY and Huang Z: Protective effects of paeoniflorin against corticosterone-induced neurotoxicity in PC12 cells. Phytother Res 26: 969-973, 2012

34. Zhang LL, Wei W, Wang NP, Wang QT, Chen JY, Chen Y, $\mathrm{Wu} \mathrm{H}$ and $\mathrm{Hu} \mathrm{XY}$ : Paeoniflorin suppresses inflammatory mediator production and regulates $G$ protein-coupled signaling in fibroblast-like synoviocytes of collagen induced arthritic rats. Inflamm Res 57: 388-395, 2008.

35. Kim ID and Ha BJ: The effects of paeoniflorin on LPS-induced liver inflammatory reactions. Arch Pharm Res 33: 959-966, 2010. 